\title{
COMUNIDADES INDÍGENAS Y AFRODESCENDIENTES: DE LA CONSTITUCIONALIZACIÓN DEL DERECHO A LA CONSULTA PREVIA ${ }^{1}$
}

\author{
Indigenous and Afro-Descendants Communities: from the \\ Constitutionalization of the Right to Prior Consultation ${ }^{1}$
}

\section{Emilio Molina Barboza²}

Fecha de Recepción: Mayo 5 de 2014

Fecha de Aceptación: Mayo 12 de 2014

SUMARIO: 1. Introducción; 2. Justificación; 3. Desarrollo; 3.1. Las Consulta previa y sus características fundamentales; 3.2. Desarrollo jurisprudencial de la Corte Constitucional frente a la protección del derecho a la consulta previa de las comunidades indígenas y afrodescendientes en Colombia; 3.2.1. El control de constitucionalidad en Colombia; 3.2.2. Derecho a la consulta previa en el marco de la jurisprudencia constitucional; 4. Conclusiones; 5. Referencias bibliográficas.

\footnotetext{
${ }^{1}$ Este trabajo es producto de un avance parcial del proyecto de investigación denominado: Protección Constitucional al Derecho a la Consulta Previa de las Comunidades Indígenas y Afrodescendientes desde la Jurisprudencia de la Corte Constitucional Colombiana. Iniciada en el país de Colombia, en la fecha de enero de 2014, y del cual participo como autor único y principal. Financiación propia.

${ }^{2}$ Candidato de a Magister en Estado de Derecho Global y Democracia Constitucional de la Universidad de Génova-Italia. Integrante del Grupo de investigación Teoría jurídica y derechos fundamentales "phrónesis" de la Universidad de Cartagena y de la Universidad Libre de Colombia-Cartagena.Email: emilio_molina09@hotmail.com
} 


\section{COMO SE CITA ESTE ARTÍCULO (APA 6)}

Molina Barboza, E. (2014) Comunidades indígenas y afrodescendientes: de la constitucionalización del derecho a la consulta previa. (Y. Carrillo De la rosa, Ed.) Revista Jurídica Mario Alario D'Filippo, VI (12), pág. $10-21$

\section{RESUMEN}

La Corte Constitucional de Colombia ha señalado que la consulta previa es un derecho fundamental, del cual son titulares los grupos étnicos del país: comunidades indígenas, afrodescendientes y gitanos. Desde esta perspectiva, resulta de gran relevancia jurídica estudiar e identificar la procedencia de la consulta previa como mecanismo de protección de los derechos de estas comunidades, en especial cuando son vulnerados en los casos de exploración y explotación de recursos naturales de sus territorios.

En esta investigación jurídica de tipo cualitativo, en la que se utilizan fichas bibliográficas como técnica de recolección de datos y el análisis de textos como método, se determinarán los derechos que protege la consulta previa y los fundamentos jurisprudenciales como requisito de procedibilidad.

\section{PALABRAS CLAVE}

Consulta previa, afectación directa, comunidad indígena, comunidad afrodescendiente, jurisprudencia constitucional.

\section{ABSTRACT}

Colombia's Constitutional Court has indicated that the consultation is a fundamental right, which are holders ethnic groups in the country: indigenous communities, Afro-descendants and Gypsies. From this perspective, it is of great legal significance to study and identify the source of the consultation as a mechanism for protecting the rights of these communities, especially when they are violated in cases of exploration and exploitation of natural resources in their territories.

In this legal qualitative research, in which bibliographic records as a technique for data collection and analysis of texts and method used, the rights protected prior consultation and jurisprudential foundations as a procedural requirement is determined.

\section{KEYWORDS}

Consultation, direct concern, indigenous community, Afro-descendant community, constitutional jurisprudence. 


\section{INTRODUCCIÓN}

El presente trabajo pretende abordar como ha sido el tratamiento jurisprudencial constitucional respecto del derecho a la consulta previa de las comunidades indígenas y afrodecendientes en Colombia, en cuanto a su protección constitucional.

La Corte Constitucional, se ha pronunciado sobre la consulta previa, a partir de análisis de casos concretos en sentencias de Acción de Tutela y en sede de Control Abstracto de Constitucionalidad, fijando las reglas que deben tenerse en cuenta para determinar la procedencia de la misma.

Ahora bien, este alto tribunal ha precisado que la Consulta previa es procedente siempre que se determine que exista una afectación directa. En este sentido entiende la Corte que hay una afectación directa cuando la medida altera el estatus de la comunidad, bien sea porque le impone restricciones o gravámenes, o, por el contrario, le confiere beneficios.

Es claro, a partir de lo anterior, que lo que debe ser objeto de consulta previa, de conformidad con la Corte Constitucional, son aquellas medidas susceptibles de afectar específicamente a las comunidades étnicas en su calidad de tales, y no aquellas disposiciones que se han previsto de manera uniforme para la generalidad de los colombianos.

Con el presente trabajo lo que se busca es identificar y establecer las reglas jurídicas desarrolladas por la jurisprudencia constitucional para poder determinar la procedencia de la consulta previa como mecanismo de protección de las comunidades indígenas y afrodescendientes.

El trabajo de investigación en curso es denominado: Protección Constitucional al Derecho a la Consulta Previa de las Comunidades Indígenas y Afrodescendientes desde la Jurisprudencia de la Corte Constitucional Colombiana, que es el fundamento de este avance investigativo, teniendo como objetivo general: Determinar el desarrollo jurisprudencial del derecho a consulta previa de las comunidades indígenas y afrodescendientes desde la jurisprudencia de la Corte Constitucional de Colombia. Y sus objetivos específicos son: (I) Estudiar el alcance del derecho a la consulta previa, conceptualización, sus elementos y su régimen jurídico. (II) Analizar los elementos y características que identifican a las comunidades Indígenas y Afrodescendientes de conformidad con en el ordenamiento jurídico colombiano. (III) Establecer la línea jurisprudencial de la Corte Constitucional frente a la protección constitucional del derecho a la Consulta Previa de las comunidades Indígenas y Afrodescendientes en Colombia.

\section{JUSTIFICACIÓN}

Este trabajo es importante y pertinente por cuanto propone realizar un abordaje sobre la protección por parte del juez constitucional, como tribunal supremo de la jurisdicción constitucional en Colombia, frente a la situación en torno a la explotación y exploración de los recursos naturales de los cuales dependen las comunidades indígenas y afrodescendientes en Colombia. 


\title{
3. DESARROLLO
}

\subsection{LA CONSULTA PREVIA Y SUS CARACTERÍSTICAS FUNDAMENTALES}

La consulta previa, es un procedimiento caracterizado por ser anterior a la toma de una decisión, o medida, de orden legislativa o administrativa susceptible de afectar la utilización, administración y conservación de los recursos naturales existentes en las tierras y territorios de los pueblos indígenas; así lo indica la doctrina:

\begin{abstract}
"Ios pronunciamientos de distintos organismos internacionales han coincidido en afirmar que esta tiene que llevarse a cabo antes de tomar la medida o realizar el proyecto que sea susceptible de afectar a las comunidades. En el caso de medidas legislativas y administrativas, si bien el Convenio 169 no ordena específicamente en cuál etapa debe llevarse a cabo la consulta, la [Comisión de Expertos en la Aplicación de Convenios y Recomendaciones] ha establecido que debe realizarse previamente a la adopción de las medidas". En el mismo sentido, el Comité de Derechos Humanos ha sostenido que la admisibilidad de las medidas que afecten a los pueblos indígenas, incluidas las legislativas, depende de que las comunidades "hayan tenido oportunidad de participar en el proceso de adopción de decisiones relativas a esas medidas". (Rodríguez \& otros, 2010, p75)
\end{abstract}

La consulta previa, es además, un proceso de concertación en el que deben discutirse los intereses del Estado frente a los intereses de grupos étnicamente protegidos constitucionalmente. De este modo la consulta previa se encuentra determinada (I) por el principio de la buena fe; a la vez, (II) debe ser informada; y (III) entendida como un medio para llegar a un acuerdo.

En relación al principio de la buena fe, este, se encuentra contemplado como un criterio mínimo de la consulta previa, de conformidad con el Artículo 60 del Convenio 169 de la OIT, el cual establece que:

"Ias consultas llevadas a cabo en aplicación de este convenio deberán efectuarse de buena fe y de una manera apropiada a las circunstancias, con la finalidad de llegar a un acuerdo o lograr el consentimiento acerca de las medidas propuestas".

De igual forma, en la Declaración de los Pueblos Indígenas, se establece que "los Estados celebrarán consultas y cooperarán de buena fe con los pueblos indígenas" antes de adoptar y aplicar medidas legislativas o administrativas y de aprobar proyectos que los afecten ${ }^{3}$.

El contenido del concepto de "buena fe", ha sido esclarecido por la Comisión de Expertos en la Aplicación de Convenios y Recomendaciones de la Organización Internacional del Trabajo. De esta manera se ha señalado que el concepto de la consulta a las comunidades indígenas comporta el establecimiento de un diálogo genuino entre ambas partes, caracterizado por la comunicación y el entendimiento, el respeto mutuo y la buena fe, con el deseo sincero de llegar a un acuerdo común

${ }^{3}$ Declaración de los Pueblos Indígenas, Arts. 19, 32. 
(CACR, 2005). De lo que se trata, es de ambientar un clima de confianza entre las comunidades indígenas y el Estado, para poder llevar a cabo un diálogo productivo.

De otro lado, al respecto del deber de ser informar la consulta previa, el derecho internacional y la doctrina, sostienen que, además del principio de buena fe, el deber de suministrar información completa a las comunidades consultadas es un criterio esencial que debe caracterizar el proceso de la consulta previa (Rodríguez \& otros, 2010, p72). La Corte Interamericana ha establecido que el deber de consultar requiere que el Estado acepte y brinde información, lo cual implica una comunicación constante entre las partes. Asimismo, ha sostenido que el Estado debe asegurarse de que los miembros de las comunidades consultadas tengan conocimiento de los posibles riesgos, incluido los riesgos ambientales y de salubridad, a fin de que acepten el plan de desarrollo o inversión propuesto con conocimiento ${ }^{4}$.

Por último y adicionalmente, en relación a la consulta previa como un medio para llegar a un acuerdo, señala el Convenio 169 de la OIT y la Declaración de los Pueblos Indígenas que, el procedimiento de la consulta previa, debe caracterizarse, también, por tener como objetivo llegar a un acuerdo u obtener el consentimiento, dependiendo de la fuente de derecho internacional que se revise ${ }^{5}$.

En todo caso, la consulta previa no debe ser concebida como un simple procedimiento por el cual se socializan algunas decisiones ya tomadas sobre una medida específica, independientemente de las opiniones que puedan tener las comunidades afectadas al respecto, sino que debe entenderse y desarrollarse como un verdadero mecanismo de consulta, participación y conciliación, cuyo propósito sea lograr un acuerdo entre las partes. (Rodríguez \& otros, 2010, p74)

\subsection{DESARROLLO JURISPRUDENCIAL DE LA CORTE CONSTITUCIONAL FRENTE A LA PROTECCIÓN DEL DERECHO A LA CONSULTA PREVIA DE LAS COMUNIDADES INDÍGENAS Y AFRODESCENDIENTES EN COLOMBIA}

A continuación nos proponemos aproximarnos a una descripción del sistema de control de constitucionalidad en Colombia, para luego puntualizar sobre el derecho a la consulta previa de las comunidades indígenas y afrodescendientes en el marco de la jurisprudencia de la Corte Constitucional Colombiana.

\subsubsection{El control de constitucionalidad en Colombia}

En Colombia el control de constitucionalidad de las leyes se encuentra a cargo de la Corte Constitucional, el cual es un órgano de naturaleza jurisdiccional. Con la entrada vigencia de la Constitución de 1991, es confeccionada, de forma inédita, la Corte Constitucional, con el propósito de erigirla como el órgano garante de la supremacía de la Constitución en el ordenamiento jurídico colombiano.

${ }^{4}$ Corte IDH, Saramaka vs. Surinam, párr. 133.

${ }^{5}$ Véase, p. ej., Convenio 169, Art. 6; Declaración de los Pueblos Indígenas, Art. 19. 
Al interior de la doctrina, se afirma, que en el caso de Colombiano, de lo que se trata es de un sistema judicial de control de constitucionalidad de carácter mixto, que comparte características de los sistemas concentrados y difuso (Quinche, 2009, p13); tal como lo ha sostenido la misma Corte Constitucional (2011), al señalar que "el sistema de control de constitucionalidad adoptado en Colombia es mixto en la medida en que combina elementos del sistema difuso y el sistema concentrado 6 .

Dentro de esta línea, por una lado, son elementos del sistema difuso los fallos emitidos por todos los jueces de la república con ocasión del ejercicio de las Acciones Constitucionales y especialmente de la Acción de Tutela, así como los que son producto de la Excepción de Inconstitucionalidad; y por otra lado, son elementos del sistema concentrado la institución de un órgano jurisdiccional -Corte Constitucional- como principal garante de la supremacía de la constitución, facultado para modular la interpretación de normas y de expulsarlas del ordenamiento jurídico, en el evento en que estas sean contrarias a la constitución. (Rey, 2008, p67)

Por último, es preciso señalar que el control judicial de constitucionalidad en Colombia, puede ser ejercido por vía de acción, y a través de controles que no requieren de la interposición de acciones. Son tres los controles por vida de acción: (I) la Acción Pública de Inconstitucionalidad; (II) la Acción Pública de Nulidad; y (III) la Acción de Tutela. Y En lo referente a los controles que no requieren de la interposición de acciones, son dos los controles: (I) a través de Control Previo; y (II) a través del Control por Vía de Excepción. (Rey, 2008, p68)

\subsubsection{Derecho a la consulta previa en el marco de la jurisprudencia constitucional}

La Corte Constitucional se ha pronunciado sobre la Consulta Previa, fijando las reglas que deben tenerse en cuenta para determinar la procedencia de la misma, a partir de análisis de casos en sentencias de tutela y en sede de control abstracto de constitucionalidad.

Recientemente la jurisprudencia constitucional, ha puntualizado aún más las reglas jurídicas de procedencia de la Consulta Previa. Tres importantes pronunciamientos merecen ser analizados, pues en los mismos se encuentra el estado actual de las reglas jurídicas que deben tenerse en cuenta para determinar los eventos en los cuales resulta obligatoria la Consulta Previa.

Para los efectos desarrollaremos la siguiente línea jurisprudencial, con el propósito de describir y analizar los diferentes argumentos y tesis sostenidas por el máximo tribunal de la jurisdicción constitucional en Colombia para garantizar la protección al derecho a la Consulta Previa de las Comunidades Indígenas y Afrodescendientes. Partiremos desde la primera de los últimos tres pronunciamientos de la Corte Constitucional respecto del derecho a la consulta previa de las comunidades indígenas y afrodescendientes ${ }^{7}$ :

\footnotetext{
${ }^{6}$ En idéntico sentido las sentencia C-836 de 2011 y SU-1219 de 2011.

${ }^{7}$ Las siguientes sentencias relacionadas en esta línea jurisprudencial pueden ser consultas en la página web de la Corte Constitucional de Colombia. http://www.corteconstitucional.gov.co/relatoria/.
} 


\section{(A) Sentencia T-698 de 2011: Elementos de procedencia de la Consulta Previa}

En el presente caso la Secretaria de Planeación del municipio de Riosucio, concedió en un predio de carácter privado, una licencia de construcción para una estación de telefonía celular de la Empresa Comcel S.A., predio que el Resguardo Indígena Cañamomo Lomaprieta, consideró hacía parte de su territorio ancestral.

Para resolver el caso, la Corte reiteró la regla constitucional de la afectación directa a comunidades étnicamente diferenciadas, como criterio determinante para establecer si es obligatoria la consulta previa a la comunidad.

En el presente caso bajo estudio, la Corte Constitucional, estableció que el título de propiedad privada para construir la estación de comunicaciones no era lo esencial para que la Consulta Previa resulte obligatoria. El criterio esencialmente determinante para identificar la procedencia de la Consulta Previa es la afectación directa a la comunidad étnicamente diferenciada en su hábitat natural.

Elaboró, además, consideraciones respecto de los aspectos probatorios a tener en cuenta para valorar la procedencia de la Consulta Previa; entre las cuales cabe destacar las siguientes: (i) la ausencia de reconocimiento formal de la existencia de territorios ancestrales o propiedad colectiva no implica que no deba adelantarse la consulta previa; (ii) La certificación de existencia de grupos étnicos en la zona donde se vaya a adelantar la medida administrativa es un indicio sobre el deber de adelantar la consulta previa, pues es un "instrumento válido al que deben acudir las autoridades y a los particulares interesados en la medida susceptible de afectar a las comunidades indígenas.

Se reiteró de igual forma que para la procedencia de la Consulta Previa debe aplicarse el criterio de afectación directa, según el cual no toda medida administrativa o legislativa genera el deber de realizar la Consulta Previa, sino solo aquellas actividades, obras o proyectos que tengan capacidad de afectar directamente a la comunidad en lo social, en lo económico, en lo cultural y en lo ambiental, mediante la modificación de su status jurídico como sujeto protegido constitucionalmente.

Teniendo en cuenta los anteriores presupuestos, se tutelaron los derechos del Resguardo Indígena Accionante, ordenando la realización de la consulta previa y la suspensión del funcionamiento de la estación de comunicaciones, por encontrarse que la misma afectaba directamente a la comunidad indígena.

\section{(B) Sentencia T-348 de 2012: Alcance Jurídico de la Consulta Previa}

El segundo pronunciamiento, es la sentencia T-348 de 2012, con ponencia de Jorge Pretelt Chaljub. Aquí la Corte resolvió el conflicto iniciado por la interposición de una acción de tutela en contra del Distrito Turístico y Cultural de Cartagena de Indias, el Consorcio Vía al Mar, el Ministerio de Ambiente y de Desarrollo, el Instituto Nacional de Concesiones y el Instituto Nacional de Vías, por parte de la Asociación de Pescadores de las Playas de Comfenalco, por cuanto no se les garantizó un 
espacio de participación y concertación previo a la construcción del proyecto "Anillo Vial - Malecón del Barrio Crespo".

La Asociación de Pescadores de las Playas de Comfenalco -ASOPESCOMFE solicita que se amparen los derechos fundamentales de sus integrantes al trabajo, a la paz, a la salud mental, a la vida digna, a la libre escogencia de profesión u oficio, a la protección especial de la tercera edad, de las minorías étnicas y de la diversidad cultural y a la dignidad humana, y en consecuencia, que se ordene al Consorcio Vía al Mar que se abstenga de encerrar el sitio donde los pescadores parquean sus botes de madera y realizan su labor de pesca, hasta tanto no se llegue a un acuerdo sobre el resarcimiento de los perjuicios que se están causando con la realización del proyecto Anillo Vial Malecón de Crespo.

Ahora bien, el Consorcio Vía al Mar mantiene y opera la carretera Cartagena - Barranquilla, ruta 90A, conocida como la vía al mar. El Consorcio tiene a cargo las labores de diseño y construcción del anillo vial de Crespo. En desarrollo de esa labor, presentó al Ministerio del Medio Ambiente y Desarrollo Sostenible la información y documentación necesaria para la expedición de la licencia ambiental.

Para el efecto, solicitó al Ministerio del Interior certificación sobre la presencia de comunidades indígenas y negras en el área de influencia del proyecto. Mediante las certificaciones OFI09-15106GCP-0201 y la OFI11-31911-GCP-201 del 14 de mayo de 2009 y del 28 de julio de 2011, respectivamente, el Ministerio del Interior certificó la no presencia de las comunidades mencionadas en la zona del proyecto.

La Corte se planteó estudiar si el Consorcio Vía al Mar y las demás entidades demandadas vulneraron los derechos fundamentales al trabajo, a la libre escogencia de oficio o profesión, a la consulta previa de la comunidad pesquera y los derechos de los adultos mayores, al omitir garantizar un espacio de participación y concertación previo a la construcción del proyecto "Anillo Vial - Malecón del Barrio Crespo" con ASOPESCOMFE, y no ofrecerle a sus miembros medidas de reparación adecuadas por los daños que el proyecto les ha causado.

Para los efectos la Corte argumento lo siguiente, respecto al alcance de la consulta previa y su fundamento:

Adelantar procesos de consulta con las comunidades indígenas y tribales para la adopción y la ejecución de decisiones que puedan afectarles, es una expresión y desarrollo, no solo de las disposiciones generales de participación ciudadana mencionadas anteriormente, sino también de las disposiciones constitucionales específicas que protegen a estas comunidades, como los Artículos 7ํ, 70, 329 y 330 de la Constitución Política, los cuales reconocen y protegen la diversidad étnica y cultural y especialmente los territorios de estas comunidades.

[La consulta previa] comprende la facultad de comunidades étnicas de determinar sus propias instituciones y estructuras, conservar sus normas, costumbres y visión del mundo y opción de desarrollo y proyecto de vida y adoptar las decisiones que se muestren más 
acordes con su cosmovisión y la protección de sus objetivos. Lo anterior tiene específicamente tres aristas: a) el derecho a participar de cualquier decisión que pueda afectarles directa o indirectamente, prerrogativa que se manifiesta en el derecho a la consulta previa y respecto al derecho general de participación; b) la participación en las decisiones políticas; $y$ c) el derecho al autogobierno de las autoridades étnicas.

No obstante a lo anterior, la Corte Constitucional, a pesar de reconocer que debía consultársele como organización que podía resultar afectada en sus actividades económicas y en su mínimo vital, no tuteló el derecho fundamental a la consulta previa, por cuanto "no es aplicable, en razón a que no se está ante un grupo culturalmente diferenciado titular de este derecho especial de participación, como son los indígenas y afrodescendientes". Sin embargo manifestó también que:

el hecho de que los pescadores no sean parte de una población identificada como indígena o afrodescendiente, no implica una autorización para que las obras de un macroproyecto se realicen ignorando al resto de la comunidad posiblemente afectada. De hecho, por tratarse de obras que pueden afectar el medio ambiente y las prácticas productivas, se debe garantizar que la comunidad en general y sobretodo del grupo de personas que depende del espacio hídrico para acceder a un ingreso mínimo, tengan conocimiento claro e integral de la obra que se realizará, así como de los efectos positivos y negativos que se prevén, y participen en el diseño de las medidas de mitigación y compensación correspondientes.

En conclusión, a pesar de que la Corte Constitucional, no amparó el derecho fundamental a la consulta previa reclamado por esta comunidad, fundamentándose en que la consulta previa es un derecho del que solo son titulares las comunidades indígenas y afrodecendientes, la Corte realiza una extensión analógica del derecho a la consulta previa, dejando en claro que a pesar de que nos encontremos frente a comunidades distintas a las indígenas o afrodescendientes, esto no es óbice para desproteger e ignorar al resto de comunidades que puedan resultar afectadas por las intervenciones del Estado en el medio ambiente. Por lo cual, en los términos de esta sentencia, se debe garantizar al resto de comunidades, el conocimiento de la obra, así como enseñar los efectos positivos y negativos que se prevén, y que participen en el diseño de las medidas de mitigación y compensación correspondientes.

\section{(C) Sentencia T-376 de 2012: Garantía de la Consulta Previa como derecho fundamental}

El último precedente sobre la materia lo constituye la sentencia T-376 de 2012 Magistrado Ponente María Victoria Calle Correa. Esta sentencia refleja el estado actual de la cuestión de la consulta previa frente a las reglas que estructuran la obligatoriedad de adelantarla, y aclara los eventos en los cuales debe obtenerse el consentimiento previo, libre e informado para establecer una medida administrativa o legislativa. El caso se refiere a la acción de tutela interpuesta en razón al otorgamiento de una concesión marítima otorgada a un particular en el sector de la Boquilla del Distrito Turístico y Cultural de Cartagena de Indias, sin haber consultado a los miembros de la comunidad afrodescendiente de la Boquilla para la imposición de la medida.

Para empezar debemos precisar que la Corte Constitucional señaló en esta sentencia que las 
disposiciones legales y reglamentarias tales como la Ley 99 de 1993 y el Decreto 1320 de 1998, son supuestos específicos previamente determinados por el Estado, en los cuales debe fundamentarse la consulta previa, pero no son los únicos determinados por el ordenamiento jurídico, habida cuenta que, lo determinante son los criterios contenidos en el Convenio 169 de la OIT desarrollados por la Jurisprudencia; y que el análisis de la misma deba hacerse en el caso concreto, esto es, mediante la aplicación de las reglas frente a las circunstancias especiales del caso.

En lo concerniente a la propiedad colectiva, estableció que es un derecho que, en principio, surge de la posesión ancestral, de lo que se desprende que su reconocimiento estatal no tiene carácter constitutivo, independientemente de su importancia para facilitar su protección jurídica. En consecuencia, la ausencia de su reconocimiento no implica la inexistencia del derecho de propiedad colectiva, y su tardanza en su reconocimiento implica una vulneración al derecho fundamental.

Frente a los ámbitos de participación distinguió tres: el de simple participación, el dado por el ámbito de participación por afectación directa a los derechos del grupo étnico diferenciado y el ámbito de la participación directa e intensa de grupos con las anteriores condiciones. Cuando la medida legislativa o administrativa afecta directamente a grupos culturalmente diferenciados, debe realizarse el procedimiento de consulta previa, antes de implementar cualquier medida legislativa y administrativa. Si la afectación es directa, pero además es intensa en la medida en que toca con las condiciones esenciales del grupo, debe obtenerse el consentimiento de la comunidad o de las comunidades para poder expedir la medida administrativa. En cambio, si la afectación es indirecta, deben brindarse espacios de participación a las comunidades en las decisiones que les puedan interesar "al menos en condiciones de igual naturaleza a los que tiene el resto de la población a disposición".

Indicó además, que de la consulta previa como derecho fundamental solo son titulares los pueblos indígenas y tribales, es decir, aquellos culturalmente diferenciados, y que por ese carácter, puede interponerse la acción de tutela para la protección frente a las amenazas y lesiones al mismo.

En cuanto a los aspectos probatorios, dejó claro que en materia de consulta previa rige el principio de libertad probatoria, así pueden tenerse en cuenta: títulos de propiedad colectivos, estudios etnográficos, reconocimiento estatal de la presencia de comunidades, pero en el caso de que no exista una declaratoria jurídica formal sobre su existencia no implica que no pueda probarse por cualquier medio probatorio; al respecto sobre este último tópico manifestó que:

"El reconocimiento estatal solamente contribuye a facilitar un medio de prueba sobre su existencia y, por lo tanto, a propiciar el acceso del grupo a los beneficios derivados de su condición, así como a los mecanismos de defensa de sus derechos fundamentales, pero no es un hecho constitutivo del derecho a la identidad cultural. La ausencia de reconocimiento o la imposición de trámites ineficaces o ineficientes para lograrlo, en cambio, comportan serias violaciones de sus derechos fundamentales. En otras palabras, como la existencia de una comunidad indígena es una cuestión material y puramente fáctica, puede probarse por cualquier medio que resulte adecuado para forjar la convicción del juez, en virtud del principio de libertad probatoria, el cual adquiere mayor 
fuerza en sede de tutela, debido a la informalidad de la acción y la prevalencia del derecho sustancial. || 73.3. En síntesis, cabe resaltar que (i) la existencia de una comunidad indígena o afrodescendiente no depende de un acto expreso de las autoridades públicas sino (ii) de los hechos constitutivos de la diversidad cultural y el auto reconocimiento del grupo. Sin embargo, (iii) el reconocimiento oficial facilita la prueba de la existencia de la comunidad ante la Administración y la jurisdicción y, por lo tanto, el acceso a los servicios del Estado y la protección de los derechos de los pueblos indígenas y afrodescendientes".

\section{CONCLUSIONES}

De acuerdo al análisis de la línea jurisprudencial desarrollada por la Corte Constitucional sobre la protección del derecho a la consulta previa de las comunidades indígenas y afrodecendientes las normas jurídicas que deben tenerse en cuenta sobre el particular son las siguientes:

1) La Corte Constitucional de Colombia, a través de su jurisprudencia, protege el derecho a la consulta previa de las comunidades indígenas y afrodescendientes, asumiendo en su interpretación jurídica, que este derecho, es una expresión y manifestación de los principios constitucionales de pluralidad, participación, respeto a la diversidad étnica y cultural, y derecho al medio ambiente.

2) Debe existir una medida administrativa o legislativa capaz de producir una afectación directa a los recursos naturales y, en general, al habitad o territorio en el que se desenvuelven cotidianamente las comunidades indígenas y afrodescendientes para que la consulta previa se traduzca en un requisito de procedibilidad.

3) Si se comprueba que la afectación es directa, deberá adelantarse el procedimiento de la Consulta Previa. Ahora bien, si además la afectación es intensa, la Administración Pública deberá no solo adelantar la consulta previa, sino que deberá actuar con el consentimiento previo, libre e informado de los grupos étnicos, pues de lo contrario la actuación de la Administración no se ajustará a los principios y derechos reconocidos y protegidos por la constitución. En cambio,

4) Si se comprueba que la afectación no es directa, sino indirecta, debe garantizarse a la población espacios de participación, junto con el resto de los ciudadanos, destinatarios de la medida. Esto es, en el trámite de la actuación administrativa, deberán brindarse espacios para que esos grupos de especial protección constitucional puedan exponer sus planteamientos frente a la adopción de la medida administrativa o legislativa.

5) La afectación directa debe sufrirla un grupo étnico culturalmente diferenciado. Solo es obligatorio adelantar la consulta previa si las agrupaciones son de especial protección constitucional (grupos indígenas o afro descendientes), es decir, grupos con características culturales diferenciadas del resto del conglomerado social. 


\section{REFERENCIAS BIBLIOGRÁFICAS}

\section{Artículos y publicaciones}

CEACR, (2005) “Observación Individual sobre el Convenio 169, Bolivia”.

QUINCHE RAMÍREZ, Manuel Fernando. "El Control de Constitucionalidad y el Control de Convencionalidad". Revista Centro de Estudio Políticos. En:

http://www.centrodeestudiospoliticos.com/descargas/1.8.pdf.

REY CLAVIJO, José Gerardo. "El control constitucional en Colombia a partir de la Constitución de 1991". VIA IURIS.

ROdRíGUEZ GARAVITO, Cesar y otros. (2010) “La Consulta Previa a los Pueblos Indígena”. Los estándares del Derecho Internacional. Bogotá: Universidad de los Andes.

\section{Disposiciones Normativas}

CONSTITUCIÓN NACIONAL de 1991.

CONVENIO 169 de la Organización Internacional del Trabajo.

DECLARACIÓN DE LAS NACIONES UNIDAS sobre los derechos de los pueblos indígenas.

Jurisprudencia Consultada

CORTE CONSTITUCIONAL. SENTENCIA T-698 de 2011

CORTE CONSTITUCIONAL. SENTENCIA T-348 de 2012

CORTE CONSTITUCIONAL. SENTENCIA T-376 de 2012 